\title{
HIGH ENERGY ISOSPIN BREAKING IN TECHNICOLOR THEORIES
}

\author{
T. APPELQUIST, T. TAKEUCHI \\ Center for Theoretical Physics, Department of Physics, Yale University, New Haven, CT 06511, USA
}

\author{
M.B. EINHORN \\ Department of Physics, University of Michigan, Ann Arbor, MI 48109, USA
}

and

\author{
L.C.R. WIJEWARDHANA \\ Department of Physics, Liniversity of Cincinnati, C'incinnati, OH 45221, LSA
}

Received 20 Septcmber 1989

\begin{abstract}
Within technicolor theories, we explore the impact of four-fermion interactions on the fermion mass splittings via explicit breaking of the custodial $\mathrm{SL}(2)_{\mathrm{R}}$ symmetry. Assuming they do not lead to unacceptably large flavor-changing neutral currents, the question is whether the large b-t quark mass splitting can be accommodated given the expcrimental limits on $\rho-1$, where $\rho \equiv M_{\mathrm{w}}^{2} / M_{\mathrm{Z}}^{2} \cos ^{2} \theta_{\mathrm{w}}$. We argue that this is possible, although further increases in the experimental lower bound on $m_{\mathrm{t}}$ may be difficult to accommodate in the present framework.
\end{abstract}

The generation of fermion mass in technicolor theories of electroweak symmetry breaking requires the introduction of new, high energy (ETC) interactions in addition to the technicolor gauge interaction [1]. These interactions generically lead to flavor-changing neutral currents (FCNC's) and must be suppressed by making the ETC interactions very weak. This can be achieved by making the associated mass scale $A$ very large compared to the technicolor confinement scale $A_{c}=\mathrm{O}(250 \mathrm{GeV})$. A hicrarchy of order $10^{3}$ between these scales is adequate for the suppression, and will be assumed for the estimates in this paper. The problem then is that the fermion masses typically turn out to be far too small, no more than a fraction of an $\mathrm{MeV}$. Walking technicolor theories [2] ${ }^{\sharp 1}$, in which the gauge coupling runs slowly

\# The idea of raising the fermion condensate was first discussed by Holdom [3]. To achicve this he imagined that the theory might have an ultraviolet stable fixed point. He also examined slowly running theories [4] to try to produce a hierarchy between the confinement and chiral breaking scales. He noted that such thcorics can also cnhance fermion masses. above the weak scale $=\mathrm{O}\left(A_{\mathrm{c}}\right)$, may provide at least a partial solution to this problem. The natural enhancement of high momentum components in these theories can elevate the technicolor chiral condensate, to which the masses of quarks and leptons are directly proportional, without substantially affecting the weak scalc itself.

In a recent letter [5], another, potentially important source of this enhancement was explored. It had previously been assumed that the dynamical symmetry breaking and technicolor condensate formation was primarily governed by the technicolor gauge interaction. The ETC interactions were then included as perturbations. In ref. [5] however, it was pointed out that if these interactions are strong enough. they can play an important direct role in the dynamical breaking and condensate formation. They must be included in the gap cquation governing the dynamical technifermion mass $\Sigma(p)$, and they can then considerably enhance its high momentum behavior and therefore the magnitude of the condensate. This effect, especially when combined with 
walking, can lead to fermion masses in the multi-GeV range and even higher.

While this is encouraging, it leads to an immediate potential problem. The ETC interactions must both be strong enough and contain enough weak isospin violation to be consistent with the lower limit on the mass of the t quark. While the ETC interactions dominantly affect the high momentum components of the theory, they also have a smaller effect on the momentum components on the order of the weak scale, which determine the $\mathrm{W}$ and $\mathrm{Z}_{0}$ masses. Even a small amount of weak isospin breaking here can pose problems if it leads to an unacceptably large deviation of the $\rho$ parameter, the ratio of the strength of the neutral- and charged-current neutrino-quark interactions, from 1 . In this letter, we shall argue that a large enough t quark mass can in fact be generated without violating the experimental constraints on the $\rho$ parameter $[6,7]$. It will be assumed throughout that there is a single ETC scale $A\left(=\mathrm{O}\left(10^{3} A_{\mathrm{c}}\right)\right)$, large enough to suppress FCNC's among the $u, d$ and $c, s$ quarks. Since the FCNC limit is much weaker among the $t$, b generation, however, there could be a smaller $\Lambda$ relevant here. If this turns out to be the casc, it will be easier to accommodate the $t$ and $b$ quarks.

The technicolor sector is assumed to consist of a set of electroweak doublets $T=(U, D)$ coupled to the technicolor gauge field. The ETC interactions are envisioned to arise from the exchange of gauge bosons of mass $A$ with couplings that respect the $\mathrm{SU}(2)_{\mathrm{L}}$ $\times U(1)$ electroweak symmetry. The effective current-current interactions among the technifermions, relcvant for $p<A$, can be constructed from the fields $L=\left(U_{\mathrm{I}}, D_{\mathrm{L}}\right)$ and $R=\left(U_{\mathrm{R}}, D_{\mathrm{R}}\right)$. The possibilities are the following:

$\bar{L}_{\gamma_{\mu}} L \bar{L} \gamma^{\mu} I$,

$\bar{L}_{\gamma_{\mu}} L \bar{R} \gamma^{\mu} R, \bar{L}_{\gamma_{\mu}} L \bar{R} \gamma^{\mu} \tau_{3} R$,

$\bar{R} \gamma_{\mu} R \bar{R} \gamma^{\mu} R, \bar{R} \gamma_{\mu} R \bar{R}_{\gamma^{\prime}} \tau_{3} R, \bar{R} \gamma_{\mu} \tau_{3} R \bar{R} \gamma^{\mu} \tau_{3} R$,

where technicolor indices and other possible indices labcling the technifermions are suppressed. There will be similar interactions coupling the technicolor sector to the quarks and leptons, and among the quarks and leptons themselves.

One could expect there to be, in addition to the interaction given in eq. (1a), an isovector interaction of the form $\bar{L}^{\alpha_{4} \gamma_{\mu} \tau} \tau L^{\alpha_{2}} \cdot \bar{L}^{\alpha_{3} \mu} \tau L^{\alpha_{1}}$ where the $\alpha_{i}$ de- note any other quantum numbers (technicolor, color, generation number) to be contracted in some way, distinguishing the various chiral doublets of the theory. Using the identity $\tau_{i j}^{\alpha} \tau_{k l}^{\alpha}=2 \delta_{i l} \delta_{k j}-\delta_{i j} \delta_{k l}$, and applying a Fierz transformation, this may be rewritten as $\bar{L}^{\alpha_{4}} \gamma_{\mu} L^{\alpha_{2}} \cdot \bar{L}^{\alpha_{3} \gamma^{\mu}{ }^{\mu}} L^{\alpha_{1}}$. Thus if we take into account that the other indices may be contracted in any order, we may assume, without loss of generality, that there are no charge-changing four-fermi interactions.

This will lead to a set of "nearly decoupled" gap equations governing the dynamical breaking for the up and down members of each weak doublet. Here we include only the $(t, b)$ doublet of ordinary quarks, coupled to itself and to a set of (U, D)'s by ETC interactions analogous to eq. (1). For the $U$ and $t$, the gap equation in ladder approximation ${ }^{\ddagger 2}$ will take the form

$$
\begin{aligned}
& \Sigma_{\mathrm{U}}(p)=\frac{1}{4} \int_{0}^{A^{2}} \frac{k^{2} \mathrm{~d} k^{2}}{M^{2}(k, p)} \frac{\alpha(M)}{\alpha_{\mathrm{c}}} \frac{\Sigma_{\mathrm{U}}(k)}{k^{2}+\Sigma_{\mathrm{U}}^{2}(k)} \\
& +\frac{\lambda_{\mathrm{UU}}}{\Lambda^{2}} \int_{0}^{1^{2}} k^{2} \mathrm{~d} k^{2} \frac{\Sigma_{\mathrm{U}}(k)}{k^{2}+\Sigma_{\mathrm{U}}^{2}(k)} \\
& +\frac{\lambda_{\mathrm{Ut}}}{\Lambda^{2}} \int_{0}^{\Lambda^{2}} k^{2} \mathrm{~d} k^{2} \frac{m_{\mathrm{t}}}{k^{2}+m_{\mathrm{t}}^{2}}
\end{aligned}
$$

where

$$
\begin{aligned}
m_{\mathrm{t}} & =\frac{\lambda_{\mathrm{l}}}{A^{2}} \int_{0}^{A^{2}} k^{2} \mathrm{~d} k^{2} \frac{\sum_{\mathrm{U}}(k)}{k^{2}+\Sigma_{\mathrm{t}}^{2}(k)} \\
& +\frac{\lambda_{\mathrm{u}}}{A^{2}} \int_{0}^{A^{2}} k^{2} \mathrm{~d} k^{2} \frac{m_{\mathrm{t}}}{k^{2}+m_{\mathrm{t}}^{2}} .
\end{aligned}
$$

The tcchnicolor running coupling is $\alpha(M)^{\sharp 3}, M(k$, $p$ ) is the maximum of $k$ and $p$, and $\alpha_{\mathrm{c}}$ is the critical technicolor coupling required for spontaneous breaking in the absence of other interactions. QCD interactions are neglected. There will be a similar equation for the $b$ quark and $D$ techniquark. In ladder approximation, only interactions of the form ( $1 \mathrm{~b}$ ) cnter, and the only mixing between the scts of equa-

\#2 Some recent work [8] indicates that the ladder approximation may provide an accurate description of chiral symmetry breaking.

*3 The Landau gauge is cmployed so that fermion wave function renormalization can be neglected to the order being considered. 
tions is through the contribution of the technifermions to the running coupling. The reliability of the four-fermion form and the use of $A$ as an ultraviolet cutoff in these equations will be addressed below.

The effective ETC couplings $\lambda_{U U}, \lambda_{U}, \lambda_{t U}$, and $\lambda_{t t}$ can be expressed in terms of the coupling strengths of eq. (1) and their $t-U$ and $t-t$ analogs. Note that the t-quark itself, through the ETC interactions, plays a role in driving the spontaneous breaking. It is also "dynamical" in this framework. This role could, a priori, be just as important as the ETC-driven technifermion contribution ${ }^{\# 4,5}$. The ETC couplings cannot be too big. In particular, $\lambda_{v u}$ and $\lambda_{\mathfrak{t}}$ must be less than unity. If they were not, then these interactions could drive the spontancous breaking by themselves, leading to a $\Sigma_{\mathrm{U}}(p)$ and $m_{\mathrm{t}}$ on the order of the ultraviolet cutoff $A$.

The $t$-quark integrals are dominated by momenta on the order of the cutoff. The technifcrmion integrals are also sensitive to cutoff physics, especially if $\Sigma_{\mathrm{U}}(k)$ falls slowly, as it will have to in order to produce a large enough value for $m_{\mathrm{t}}$. In the case of the $\mathrm{t}$ quark, all the cutoff sensitivity can be integrated out and absorbed into the $\lambda$ 's. With $m_{1} \ll \Lambda$,

$$
\begin{aligned}
& \Sigma_{\mathrm{U}}(p)=\frac{1}{4} \int_{0}^{A^{2}} \frac{k^{2} \mathrm{~d} k^{2}}{M^{2}(k, p)} \frac{\alpha(M)}{\alpha_{c}} \frac{\Sigma_{\mathrm{U}}(k)}{k^{2}+\Sigma_{\mathrm{U}}^{2}(k)} \\
& +\frac{\lambda_{\mathrm{U}}}{A^{2}} \int_{0}^{A^{2}} k^{2} \mathrm{~d} k^{2} \frac{\Sigma_{\mathrm{U}}(k)}{k^{2}+\Sigma_{\mathrm{U}}^{2}(k)},
\end{aligned}
$$

where $\lambda_{U}$ is the effective four-fermion coupling among the technifermions. All physics at the cutoff and beyond has now been absorbed into $\lambda_{\mathrm{U}}$, which must also be less than unity [5].

The program is to solve eq. (3) (as well as its D counterpart) and to note that with a sufficiently strong $\lambda_{U}<1$, the technifermion condensate $\langle\bar{U} U\rangle$ (the integral expression in the second term of eq. (3)) can be greatly enhanced relative to its naive value (of $\left.\operatorname{order} A_{\mathrm{c}}^{3}\right)$. The $\mathrm{t}$-quark mass will then be given by

$m_{\mathrm{t}}=\frac{\lambda_{\mathrm{t}}}{A^{2}} \int_{0}^{A^{2}} k^{2} \mathrm{~d} k^{2} \frac{\Sigma_{\mathrm{U}}(k)}{k^{2}+\sum_{\mathrm{U}}^{2}(k)}$,

\$4 The explicit role of the t quark was suppressed in ref. [5].

\#5 Miranski et al. [9] have recently considered the extreme possibility that a four-fermion interaction of the t quark could be entircly responsible for electroweak symmetry breaking. where $\lambda_{1}$ is the cffective $t-U$ four-fermion coupling. If $\lambda_{\mathrm{U}}$ and $\lambda_{\mathrm{t}}$ are roughly the same, then $m_{\mathrm{t}}$ will be about the same as the second term in eq. (3). Since this term can be rewritten as $\left[1+\alpha(\Lambda) / 4 \alpha_{\mathrm{c}} \lambda_{\mathrm{u}}\right]^{-1} \Sigma(\Lambda)$, it can be expected to be roughly the technifermion mass at the cutoff. Since $\lambda_{t}$ includes a sum over technicolor not necessarily present in $\lambda_{\mathrm{U}}$, however, it might naturally be several times larger than $\lambda_{U}$, These remarks apply to $\lambda_{\mathrm{D}}$ and $\lambda_{\mathrm{b}}$ as well, so that $m_{\mathrm{t}}$ and $m_{\mathrm{b}}$ might be expected to be several times larger than $\Sigma_{\mathrm{U}}(\Lambda)$ and $\Sigma_{\mathrm{D}}(\Lambda)$ respectively.

The question then is the following. If $\lambda_{\mathrm{U}}, \lambda_{\mathrm{t}}$ and $\lambda_{\mathrm{D}}$, $\lambda_{\mathrm{b}}$ are such as to give, say $m_{\mathrm{l}}>60 \mathrm{GcV}$ and $m_{\mathrm{b}} \cong 5$ $\mathrm{GeV}$, how much weak isospin breaking is induced in the technifermion masses at lower encrgies and how badly does this infect the $\rho$ parameter? The results of a numerical solution of eq. ( 3 ) and the corresponding equation for $\Sigma_{\mathrm{D}}(p)$ are shown in table $1^{\# 6}$. The fourth column gives the results for $\Sigma_{\mathrm{i}}(A)$ and $\Sigma_{\mathrm{D}}(A)$, the value of the technifermion masses at the ETC scale. While specific choices of technicolor gauge theory parametcrs are made for the computation, the results as a function of $\lambda_{U}$ and $\lambda_{\mathrm{D}}$ do not depend sensitively on the choicc. The table shows the expected result that the four-fermion interactions have a much more dramatic effect on the high momentum components $(\Sigma(\Lambda))$ than on the low momentum components $(\Sigma(0))$. In particular, with the values of $\lambda_{\mathrm{U}}$ and $\lambda_{D}$ shown, large and rather different values of $\Sigma_{\mathrm{U}}(\Lambda)$ and $\Sigma_{\mathrm{D}}(\Lambda)$ emerge while $\Sigma_{\mathrm{U}}(0) / \Sigma_{\mathrm{D}}(0)$ remains close to unity. It is only for the very largest values of $\lambda_{U}$ and $\lambda_{D}$ that this ratio begins changing substantially.

We next estimate the shift in the $\rho$ parameter due to the wcak isospin violation in the ETC interactions. The most familiar source of this shift comes from loops of ordinary quarks and leptons, whose weak isospin-violating masses arise in the present context from the ETC interactions. These contributions involve integrations that converge rapidly at momentum scales above the larger fermion mass in each weak doublet, and have been computed by several authors [11]. The results can be expressed in terms of the fermion masses, with no further sensitivity to the ETC or technicolor dynamics. The contribution of the $t, b$

\#6 The numerical results of this table and of ref. [5] can be elucidated by an analytic study as well. 
Table 1

Results of a numerical calculation of the shift in the $\rho$-parameter for various values of $\lambda_{\mathrm{U}}$ and $i_{\mathrm{D}}$. The technicolor coupling is chosen to be $\alpha(p)=\alpha(A) /[1+b \alpha(A) \ln (p / A)]$ for $A_{c} \leqslant p \leqslant A$, and $\alpha(p)=\alpha\left(A_{c}\right)$ for $0 \leqslant p \leqslant A_{c}$. Shown are the two cases $A=1 / b \alpha_{c}=2.8$ and $A=1.2$. The $A=2.8$ case corresponds to an SU(4) technicolor theory with 16 flavors of technifcrmions in the fundamental representation, while the $A=1.2$ case corresponds to the same except with 8 flavors. The factor $N$ in eqs. (5) and (6) for these theories is $N=64$ and $N=32$ respectively. The estimates for $A, \Sigma_{\mathrm{U}, \mathrm{D}}(0)$, and $\Sigma_{\mathrm{U}, \mathrm{D}}(A)$ were obtained by normalizing $F_{0}$ to be $250 \mathrm{GeV}$.

\begin{tabular}{|c|c|c|c|c|c|c|c|c|c|c|}
\hline$A$ & $\frac{\alpha(A)}{\alpha_{c}}$ & $\frac{A}{A_{\mathrm{c}}}$ & $i_{D}$ & $i u$ & $\begin{array}{l}A \\
{[\mathrm{TeV}]}\end{array}$ & $\begin{array}{l}\Sigma_{\mathrm{D}}(0) \\
{[\mathrm{GeV}]}\end{array}$ & $\begin{array}{l}\Sigma_{\mathrm{U}}(0) \\
{[\mathrm{GeV}]}\end{array}$ & $\begin{array}{l}\Sigma_{\mathrm{D}}(A) \\
{[\mathrm{MeV}]}\end{array}$ & $\begin{array}{l}\Sigma_{\mathrm{U}}(A) \\
{[\mathrm{MeV}]}\end{array}$ & $\Delta \rho$ \\
\hline \multirow[t]{5}{*}{2.8} & 0.33 & 2000 & 0.0 & 0.1 & 680 & 340 & 340 & 0.2 & 0.5 & $4 \times 10^{-10}$ \\
\hline & & & 0.6 & 0.7 & 680 & 340 & 340 & 6.0 & 13 & $2 \times 10^{-7}$ \\
\hline & & & 0.797 & 0.808 & 630 & 310 & 350 & 120 & $1.3 \times 10^{3}$ & $0.4 \%$ \\
\hline & & & 0.805 & 0.8088 & 560 & 290 & 360 & 330 & $3.0 \times 10^{3}$ & $1.5 \%$ \\
\hline & & & 0.8088 & 0.8095 & 280 & 180 & 330 & $1.5 \times 10^{3}$ & $15 \times 10^{3}$ & $7.4 \%$ \\
\hline \multirow[t]{5}{*}{1.2} & 0.18 & 500 & 0.0 & 0.1 & 420 & 440 & 440 & 0.2 & 0.8 & $8 \times 10^{-11}$ \\
\hline & & & 0.6 & 0.7 & 420 & 440 & 440 & 9.6 & 17 & $1 \times 10^{-8}$ \\
\hline & & & 0.87 & 0.895 & 410 & 430 & 450 & 140 & $1.7 \times 10^{3}$ & $4 \times 10^{-4}$ \\
\hline & & & 0.895 & 0.8973 & 340 & 380 & 490 & $1.4 \times 10^{3}$ & $15 \times 10^{3}$ & $1.6 \%$ \\
\hline & & & 0.8971 & 0.8976 & 200 & 260 & 470 & $4.9 \times 10^{3}$ & $52 \times 10^{3}$ & $5.8 \%$ \\
\hline
\end{tabular}

doublet, in the limit $m_{\mathrm{t}} \gg m_{\mathrm{t}}$, takes the form $\delta \rho=$ $\rho-1 \approx 3 m_{t}^{2} / 16 \pi^{2} F^{2}$, where $F$ is the weak scale $=250$ $\mathrm{GcV}$. From this result, it can be seen that the t-quark mass could be well above the current lower bound and still keep $\delta \rho<10^{-2}$ as required by cxperiment. Thus, for these contributions, there is no immcdiate problem keeping the $\rho$ parameter in line.

The contribution of the technicolor sector to the $\rho$ parameter is more difficult to estimate. Our procedure is to note that $\rho$ can be written in the form $\rho=$ $F_{ \pm}^{2} / F_{0}^{2}$, where $F_{ \pm}$and $F_{0}$ are the Goldstone boson decay constants in the charged and neutral channels, and then to estimate $F_{:=}$and $F_{0}$ in terms of $\Sigma_{\mathrm{U}}(p)$ and $\Sigma_{\mathrm{D}}(p)$, which have already been computed. Sum rules relating $F_{=}$and $F_{0}$ to $\Sigma_{\mathrm{U}}(p)$ and $\Sigma_{\mathrm{D}}(p)$ can be derived in various ways $[9,12-14] \# 7$. Using the approximation $\quad \Delta \rho \equiv F_{ \pm}^{2} / F_{0}^{2}-1 \approx\left(F^{2}-F_{0}^{2}\right) / F^{2}$ where $F^{2} \approx F_{ \pm}^{2} \approx F_{0}^{2} \cong(250 \mathrm{GeV})^{2}$, an expression for $F_{t}^{2}-F_{0}^{2}$ is needed. We find [14]

$$
\begin{aligned}
& F^{2}=-F_{0}^{2}=\frac{N}{128 \pi^{2}} \int_{0}^{\infty} \mathrm{d} k^{2} k^{2} \operatorname{Tr}\left[S_{D} \gamma_{\mu}-\frac{1}{2}\left(1-\gamma_{S}\right) S_{D} \widetilde{\Gamma}_{0}^{\mu}\right. \\
& \left.+S_{U \gamma_{\mu}} \frac{1}{2}\left(1-\gamma_{s}\right) S_{U^{\prime}} \widetilde{\Gamma}_{b}^{\mu}-2 S_{D} \gamma_{\mu} \cdot \frac{1}{2}\left(1-\gamma_{s}\right) S_{U^{\prime}} \widetilde{\Gamma}_{ \pm}^{\mu}\right],
\end{aligned}
$$

\$7 Ref. [14] will include a discussion of wavefunction renormalization which is set to unity in eq. (6). where $N$ counts the number of technifermions and $\widetilde{\Gamma}_{0}^{\mu}$ and $\widetilde{\Gamma}_{ \pm}^{\mu}$ are the technifermion vertex functions with the Goldstone poles removed.

These vertex functions can get contributions from the technicolor and ETC interactions. Neglecting the latter for the moment, the high momentum component part of the above integral can be estimated by using the asymptotic freedom of the technicolor interactions to make the replacement $\tilde{\Gamma}_{ \pm}^{\mu}, \tilde{\Gamma}_{0}^{\mu} \rightarrow$ $\gamma^{\mu} \cdot \frac{1}{2}\left(1-\gamma_{s}\right)$. Then using $S_{\mathrm{L}}^{-1}=k-\Sigma_{\mathrm{U}}(k), S_{\mathrm{D}}^{-1}=$ $k-\Sigma_{\mathrm{D}}(k)[14]$,

$F^{2}-F_{0}^{2} \cong \frac{N}{32 \pi^{2}} \int_{0}^{\infty} \mathrm{d} k^{2} k^{4}\left(\frac{1}{k^{2}+\Sigma_{\mathrm{U}}^{2}}-\frac{1}{k^{2}+\Sigma_{\mathrm{D}}^{2}}\right)^{2}$.

This integral expression is highly convergent for $k>\Sigma_{\mathrm{v}}(k), \Sigma_{\mathrm{v}}(k)$ making the above use of the bare vertex functions not reliable in detail. We nevertheless use cq. (6) with the caveat that it can be expected to give no more than a rough estimate in the face of the strong technicolor interactions that set in where the integral appears to be dominated. Numerical evaluation for each value of $\lambda_{\mathrm{U}}$ and $\lambda_{\mathrm{D}}$ then gives the cstimates for $\Delta \rho$ shown in the last column of table 1 . As expected, it is only for the largest $\lambda_{U}, \lambda_{D}$ values that $\rho$ begins to deviate from unity by more than the (2-3) $\%$ allowed by experiment [6] (cf. ref. [7]). 
The table indicates that if $\lambda_{\mathrm{L}}$ and $\lambda_{\mathrm{D}}$ are chosen to give $\Sigma_{\mathrm{D}}(\Lambda) \approx m_{\mathrm{b}}$ and $\Sigma_{\mathrm{U}}(\Lambda)>60 \mathrm{GeV}$, the $\Delta \rho$ estimate begins to become uncomfortably large. Even taking into account that eq. (6) is a crude estimate, it looks as though this is too much weak isospin violation. It is the presence of the technicolor interactions that make the technifermion contributions more sensitive to weak isospin breaking than the $t$, $b$ contribution. These interactions generate an anomalous dimension $\left(=-3 C_{2} / 2 \pi b\right)$ for the $\mathrm{U}$ and $\mathrm{D}$ mass operators, leading to a larger value for $\Sigma_{\mathrm{U}}(0)-\Sigma_{\mathrm{D}}(0)$ than for $\Sigma_{U}(\Lambda)-\Sigma_{\mathrm{D}}(\Lambda)$. It is interesting to note that slow running enhances this effect at the same time that it makes it easier to produce larger fermion masses.

Of course, it is not likely that $\Sigma_{\mathrm{U}}(\Lambda)$ and $\Sigma_{\mathrm{D}}(A)$ need to be taken as large as $m_{\mathrm{t}}$ and $m_{\mathrm{b}}$. As pointed out above, it is natural to assume that $\lambda_{t}, \lambda_{b}$ are several times larger than $\lambda_{\mathrm{U}}, \lambda_{\mathrm{D}}$. If that is the case, $m_{\mathrm{t}}$ and $m_{\mathrm{b}}$ can be accommodated with smaller values of $\Sigma_{\mathrm{U}}(\Lambda)$ and $\Sigma_{\mathrm{D}}(\Lambda)$-values that keep $\Delta \rho$ acceptable. It is also worth noting that we have assumed here that the entirc $F^{2} \cong(250 \mathrm{GeV})^{2}$ must bc made up of the technifermions that couple to the $\mathbf{t}-\mathbf{b}$ generation. It could be, on the other hand, that a sizeable fraction of $F^{2}$ is built up from additional technifermions that couple only to the $u, d$ and $c, s$ generations. Since these will involve a smaller $U-D$ mass splitting, they will tend to reduce the effect of the technifermions of the $\mathrm{t}-\mathrm{b}$ generation on the $\rho$ paramcter.

These estimates suffer from many uncertainties, such as those associated with the strong technicolor interactions. What seems clear, nevertheless, is that the accommodation of the $t$ quark within this framework is possible but difficult. Further increases in the lower bound on $m_{1}$ may not be tolerable without some new ingredient. We have, of course, been very conservative in taking the ETC scale $A$ associated with the $t, b$ generation to be the same as that associated with the lighter quarks. If it is lowered, the t- and bquark masses can more easily be generated without exceeding the experimental upper limit on $\Delta \rho$.

Finally, we comment on the role of four-fermion ETC interactions in the vertex functions in eq. (5). The simplest possibility is the appearance of a single ETC vertex, arising from eq. (lc) for examplc, lcading to a "figure eight" contribution to the $\rho$-parameter. This sort of contribution was in fact discussed several years ago [15]. It is not difficult to see that in the present context, with $A \gg \Lambda_{\mathrm{TC}}$, this will lead to a negligibly small contribution to the $\rho$-parameter. The integrals in the figure eight are sufficiently convergent that the factor of $A^{-2}$ cannot be compensated. The suppression of the ETC contribution is, in fact, a general feature. The four-fermion, and higher-dimension, opcrators, since they respect the symmetrics of the renormalizable gauge interactions, will lead cither to physical effects suppressed at low energies, or to unobservable renormalizations of the gaugc theory couplings. The dynamical fermion masses partly "evade" this decoupling of the ETC physics, through the large condensate enhancements driven by these interactions along with the renormalization effects of the technicolor interactions for $p \ll A$. It is these contributions that are being roughly estimated by eq. (6). A future paper [14] will provide a more detailed description of decoupling and spontaneous chiral symmetry breaking.

While it is possible to pick a set of $\lambda$ 's to fit the various quark masses, and to satisfy the experimental constraints on the $\rho$ parameter, it remains an open question whether these values will emerge from an underlying theory. The table shows that the fermion masses, especially the larger ones, are very sensitive to small changes in the $\lambda$ 's. Whether this is an example of unnatural fine tuning or a hint at the correct solution to the fermion mass hierarchy problem remains to be seen.

Helpful discussions with M. Soldate, B. Holdom, Y-P. Yao and S. Chivukula are gratefully acknowledged. One of us (M.B.E.) would like to acknowledge the support of the Centre de Physique Theorique, CNRS Luminy, where a portion of this work was carricd out, and to thank J. Soffer for his hospitality there. This work is supported in part by the US Department of Energy under contract number DEAC02-ERO-3075 and by an O.J.I. Grant of the DOE through the contract FG-0284-ER-40153; Michigan DOE contract number DE-AC02-76ERO1112.

\section{References}

[1] S. Dimopoulos and L. Susskind, Nucl. Phys. B 155 (1979) 237;

E. Eichten and K. Lanc, Phys. Lett. B 90 (1980) 125. 
[2] T. Appelquist, D. Karabali and L.C.R. Wijewardhana, Phys. Rev. Lett. 57 (1986) 957;

T. Appelquist and L.C.R. Wijewardhana, Phys. Rev. D 35 (1987) 774; D 36 (1987) 568.

[3] B. Holdom, Phys. Rev. D 24 (1981) 1441.

[4] B. Holdom, Phys. Lett. B 150 (1985) 301.

[5] T. Appelquist, M. Einhorn, T. Takeuchi and L.C.R. Wijewardhana, Phys. Lett. B 220 (1989) 223.

[6] For a recent review, see P. Langacker, Invited talk XXIV Intern. Conf. on High energy physics (Munich, August 1988); and 1988 Mecting of the Division of Particles and Fields of the APS (Storrs, CT, August 1988).

[7] For a related analysis see R.S. Chivukula, Phys. Rev. Lett. 61 (1988) 2657.

[8] T. Appelquist, K. Lane and U. Mahanta, Phys. Rev. Lett. 61 (1988) 1553;
U. Mahanta, Phys. Rev. Lett. 62 (1989) 2349.

[9] V. Miranski, M. Tanabashi and K. Yamawaki, Mod. Phys. Lett. A 4 (1989) 1043.

[10] T. Takeuchi, Yale preprint YCTP-P9-89, submitted to Phys. Rev.

[11] M. Einhorn, D.R.T. Jones and M. Veltman, Nucl. Phys. B 191 (1981) 146, and references thercin.

[12] R. Jackiw and K. Johnson, Phys. Rev. D 8 (1973) 2386; H. Pagels and S. Stokar, Phys. Rev. D 20 (1979) 2947.

[13] B. Holdom, University of Toronto preprint UTPT-89-18 (May 1989).

[14] T. Appelquist, M.B. Einhorn, T. Takeuchi and L.C.R. Wijewardhana, manuscript in preparation.

[15] T. Appelquist, M. Bowick, E. Cohler and A. Hauser, Phys. Rev. D 31 (1985) 1676. 